\title{
Cold gas in dark matter halos and the formation of late-type galaxies
}

\author{
H. J. Mo ${ }^{1}$, Xiaohu Yang ${ }^{1}$, \\ Frank C. van den Bosch ${ }^{2}$ and Neal S. Katz ${ }^{1}$ \\ ${ }^{1}$ Department of Astronomy, University of Massachusetts, Amherst, MA 01003-9305, USA \\ ${ }^{2}$ Department of Physics, Swiss Federal Institute of Technology, CH-8093, Zurich, Switzerland
}

\begin{abstract}
We use recent observations of the HI mass function to constrain the amount of cold gas in dark matter halos. It is found that the cold gas mass in a halo decreases rapidly with decreasing halo mass for low-mass halos with $M<10^{12} h^{-1} \mathrm{M}_{\odot}$. This result is in conflict with the standard model, in which most of the gas in a low-mass halo is assumed to settle into a gaseous disk, and the cold gas is depleted by star formation and supernova-driven outflow until the disk becomes gravitationally stable. Heating by the UV background can reduce the amount of cold gas in halos with masses $<10^{10} h^{-1} \mathrm{M}_{\odot}$, but is insufficient to explain the observational result. A consistent model can be found if low-mass halos are embedded in a preheated medium, with a specific gas entropy $\sim 10 \mathrm{keV} \mathrm{cm}{ }^{2}$. Such a model can also explain why the faint-end slope of the galaxy luminosity function is flat. We propose a preheating model in which the medium around low-mass halos is preheated by gravitational pancaking. Since large-scale tidal fields tend to suppress the formation of low-mass virialised halos while promoting the formation of pancakes, the formation of massive pancakes may precede that of low-mass dark halos at the present time. 'Previrialisation' of such pancakes can heat the intergalactic medium. The preheated gas has a cooling time longer than the age of the Universe at $z \lesssim 2$, and has a specific entropy comparable to that required to reduce the amount of cold gas and star formation activity in galaxy halos.
\end{abstract}

\section{Introduction}

The cold dark matter (CDM) model of structure formation has been proven to be a very successful paradigm to understand the large-scale structure of the Universe. However, as far as galaxy formation is concerned, a number of important issues still remain. For example, CDM models generically predict a large number of low-mass dark matter halos, with the halo mass function $n(M)$ going with halo mass roughly like $n(M) \propto M^{-2}$ at the low-mass end, while the observed luminosity function of galaxies, $\Phi(L)$, has a rather shallow shape at the faint end, with $\Phi(L) \propto L^{-1}$. Thus, the efficiency of star formation in dark mater halos must be a strongly nonlinear function of halo mass (e.g. Yang et al. 2003; van den Bosch et al. 2003). One of the most challenging problems in modern cosmology is to understand this nonlinear relation in terms of how gas is assembled into dark halos to form stars. A number of physical processes have been proposed to explain the star formation efficiency in low-mass halos. One is that photoionisation heating by the UV background may prevent gas from cooling in low-mass halos. However, numerical simulations have shown that this effect is efficient only in dark mater halos with $M \lesssim 10^{10} h^{-1} \mathrm{M}_{\odot}$ (e.g. Gnedin 2000; Hoeft et al. 2005). Energy feedback from supernova explosions associated with star formation has also been proposed as a possible process to suppress gas cooling and star formation in low-mass halos (e.g. Dekel \& Silk 1986). However, although the total energy associated with supernova explosions in a galaxy can be larger than the total binding energy of the gas in the galaxy, it is unclear how efficient the supernova energy can be used to eject gas and to suppress star formation. 
Semi-analytical models of galaxy formation have shown that the faint-end luminosity function can be reproduced in the standard $\Lambda \mathrm{CDM}$ model, if the feedback efficiency in low-mass halos is sufficiently high (e.g. Benson et al. 2003; Kang et al. 2005). Unfortunately, such high efficiency is not supported by numerical simulations (e.g. Mac Low \& Ferrara 1999).

So far, theoretical modelling has been focused mainly on reproducing the luminosity function of galaxies, and much less attention has been paid to the cold gas in galaxies. This is partly due to the fact that cold gas is more difficult to observe, and large complete samples are more difficult to construct. The situation has improved in recent years, as a number of relatively large blind 21-cm surveys have been completed (e.g. Zwaan et al. 2005 , and references therein). With these large surveys, it is now possible to estimate accurately the cold gas mass function over a large mass range. Such observations constrain galaxy formation in a way complementary to the galaxy luminosity function, because galaxy formation is a process involving both cold gas and stars.

In this presentation, I will summarise the results of our recent analysis using observations of the HI-mass function of galaxies to constrain galaxy formation in dark halos (Mo et al. 2005). I will show that current observational data can already provide stringent constraints.

\section{Cold gas in dark matter halos}

We used the results of the HI-mass function of galaxies obtained by Rosenberg \& Schneider (2002, hereafter RS) and Zwaan et al. (2005, hereafter Z05). Both of these results were obtained from blind 21-cm surveys of HI gas. These two HI-mass functions (plotted in Fig. 1) are consistent with each other at the massive end, but there is significant difference at the low-mass end. Fitting the HI-mass function with a Schechter function, $\Theta\left(M_{\mathrm{HI}}\right)=\Theta_{\star}\left(M_{\mathrm{HI}} / M_{\mathrm{HI} *}\right)^{\beta} \exp \left(-M_{\mathrm{HI}} / M_{\mathrm{HI} *}\right)$, RS obtained a low-mass end slope $\beta=-1.53 \pm 0.05$, while Z05 obtained a shallower slope, $\beta \sim-1.37 \pm 0.03$ (see Zwaan et al. 2003 for a detailed discussion of previous HI mass function measurements). We converted the observed HI mass into a total cold gas mass by multiplying the HI mass by a factor of 1.4 to account for helium and metals. We do not include molecular gas in our budget, because the fraction of gas in molecular form is in general quite small for the galaxies considered here (e.g. Garnett 2002).

In order to link the HI mass of a galaxy to the mass of its host dark halo, we make the assumption that each of the observed HI galaxies is associated with a dark halo. This assumption may be valid, because the galaxies selected by blind 21-cm surveys are dominated by late-type galaxies. Some of the low-HI mass systems may be associated with satellite galaxies in massive halos, and so our assumption may overestimate the amount of cold gas associated with isolated low-mass halos. However, as we will see below, if our assumption is an overestimate, then the real discrepancy between model and observation is even larger.

With the above assumption, we can write the HI mass function $\Theta\left(M_{\mathrm{HI}}\right)$ in terms of the halo mass function $n(M)$ as

$$
\Theta\left(M_{\mathrm{HI}}\right)=\int P\left(M_{\mathrm{HI}} \mid M\right) n(M) \mathrm{d} M,
$$

where $P\left(M_{\mathrm{HI}} \mid M\right)$ is the distribution of $M_{\mathrm{HI}}$ for a given halo mass $M$. For the standard $\Lambda$ CDM model, $n(M)$ is well known. The observed HI mass function can therefore be used to constrain $P\left(M_{\mathrm{HI}} \mid M\right)$. However, if we do not impose any condition on the form of $P\left(M_{\mathrm{HI}} \mid M\right)$, it is impossible to obtain any stringent constraints on $P\left(M_{\mathrm{HI}} \mid M\right)$. We adopt 


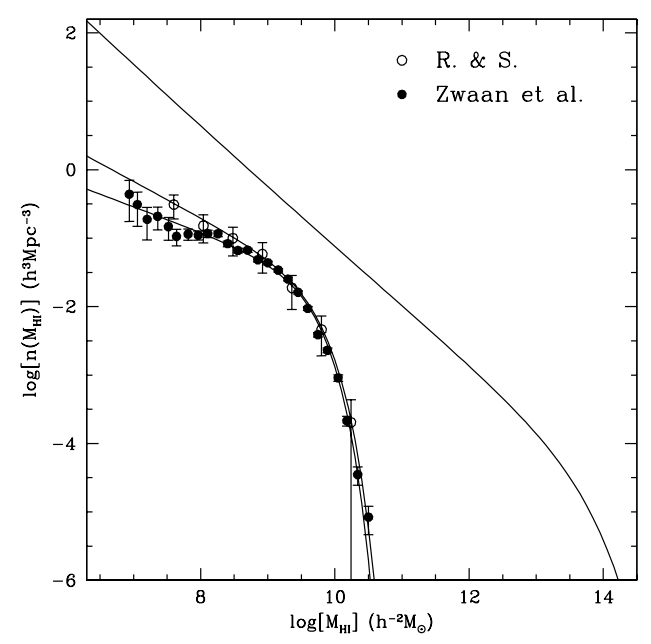

Figure 1. The observed HI mass functions by Rosenberg \& Schneider (2002) (open circles) and Zwaan et al. (2005) (solid dots), along with their Schechter function fits. The high solid curve shows the halo mass function predicted by the standard $\Lambda$ CDM model, with the halo masses scaled down by a factor equal to the universal baryon fraction of the Universe.

a simple model, in which the HI mass has a log-normal distribution,

$$
P\left(M_{\mathrm{HI}} \mid M_{h}\right) d M_{\mathrm{HI}}=\frac{1}{\sqrt{2 \pi} \sigma_{\mathrm{HI}}} \exp \left(-\frac{\ln ^{2} x}{2 \sigma_{\mathrm{HI}}^{2}}\right) \mathrm{d} \ln x,
$$

where $x \equiv M_{\mathrm{HI}} / \bar{M}_{\mathrm{HI}}$. In general, both the median $\bar{M}_{\mathrm{HI}}$ and the dispersion $\sigma_{\mathrm{HI}}$ depend on halo mass $M$. In a simple model, we assume the relation between $\bar{M}_{\mathrm{HI}}$ and $M$ is a broken power law:

$$
\bar{M}_{\mathrm{HI}}(M)=M_{\mathrm{HI}, 0} \frac{\left(M / M_{0}\right)^{\gamma_{1}}}{1+\left(M / M_{0}\right)^{\gamma_{1}-\gamma_{2}}} .
$$

We apply the above relation for halos in the mass range from $10^{8}$ to $10^{12.5} h^{-1} M_{\odot}$. We also assume $\sigma_{\mathrm{HI}}$ is independent of $M$, but we change the value of $\sigma_{\mathrm{HI}}$ to test its effect on our results. Over a large range of halo mass, the halo mass function is roughly a power law, and the assumption on $\sigma_{\mathrm{HI}}$ does not have significant impact on the $\bar{M}_{\mathrm{HI}}-M$ relation for $M_{\mathrm{HI}}<M_{\mathrm{HI}}^{*}$.

We use the Monte-Carlo Markov Chain (MCMC) method to obtain the free parameters in our model. The MCMC was implemented in the same way as in van den Bosch et al. (2005). The left panel of Fig. 2 shows the $\bar{M}_{\mathrm{HI}}-M$ relation obtained from the two HI mass functions. The constraints from the RS data are less stringent, because the sample is smaller and the errors in the data are larger. The sharp break at $M \sim 10^{11.5} h^{-1} \mathrm{M}_{\odot}$ is required by the relatively shallow shape of the $\mathrm{HI}$ mass function as compared to the halo mass function at the low-mass end. We have changed the value of $\sigma_{\mathrm{HI}}$ from 0 to 0.5 , and found that the $\bar{M}_{\mathrm{HI}}-M$ relation at $M<10^{11} h^{-1} \mathrm{M}_{\odot}$ is quite independent of the value of $\sigma_{\mathrm{HI}}$.

\section{Constraining the formation of late-type galaxies}

In small halos with $M<10^{11} h^{-1} \mathrm{M}_{\odot}$, radiative cooling of the halo gas is efficient. Thus, in the absence of heating, most of the gas in such a halo can cool and settle into a gaseous disk supported by rotation. In the standard model of star formation in disk galaxies, star 

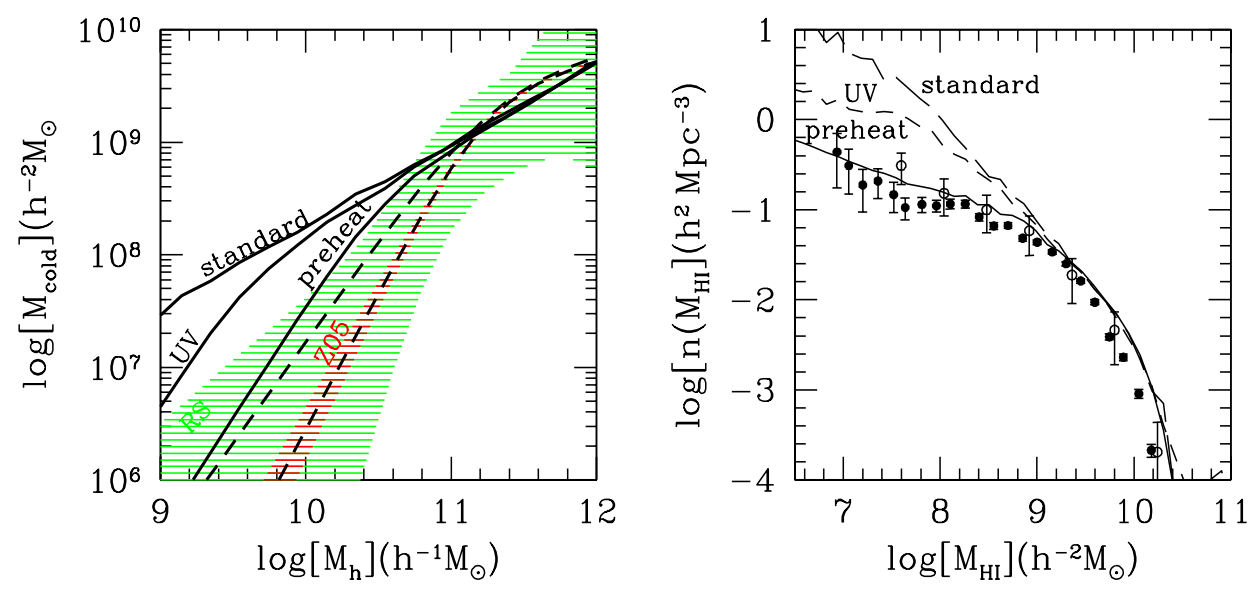

Figure 2. Left panel: the average cold gas mass in dark halos of a given mass as a function of halo mass. The darkly shaded area shows the $90 \%$ range obtained from fitting the HI mass function of Z05, while the lightly shaded area shows the same result obtained from the HI mass function of RS. Dashed curves are the corresponding medians. The predictions of the standard model, the model with heating by the UV background, and the preheating model are shown as labelled curves. Right panel: the HI-mass function predicted by the standard model (long-dashed curve), the model with heating by the UV background (short-dashed curve), and the preheating model (solid curve). The observational results of RS and Z05 are shown as the open circles and solid dots, respectively.

formation is assumed to follow the Kennicutt relation, $\Sigma_{\mathrm{SFR}}=A \Sigma_{\text {cold }}^{N}$, where $\Sigma_{\mathrm{SFR}}$ is the surface density of star formation rate, $\Sigma_{\text {cold }}$ is the surface density of cold gas, $N \sim 1.5$ and $A \sim 2.5 \times 10^{-4}$ if $\Sigma_{\text {cold }}$ is in units of $\mathrm{M}_{\odot} \mathrm{pc}^{-2}$ and $\Sigma_{\mathrm{SFR}}$ in $\mathrm{M}_{\odot} \mathrm{yr}^{-1} \mathrm{kpc}^{-2}$ (Kennicutt 1998). The cold gas in a disk is assumed to be depleted by star formation until its surface density reaches a critical value, below which the disk is gravitational stable and star formation rate drops sharply. According to Toomre's instability criterion, the critical surface density can be written as $\Sigma_{\text {crit }}=\sigma_{\text {gas }} \kappa /\left(\pi G Q_{\text {crit }}\right)$, where $\kappa$ is the local epicycle frequency and $\sigma_{\text {gas }}$ is the velocity dispersion of the cold gas. Based on observations of local star forming galaxies, Kennicutt $(1989,1998)$ obtained $\sigma_{\text {gas }} \sim 6 \mathrm{kms}^{-1}$ and $Q_{\text {crit }} \sim 1.5$.

It has been realised since the late 1970s that the star formation efficiency in lowmass halos has to be strongly suppressed in CDM models, because otherwise the theory predicts too many faint galaxies (White \& Rees 1978). A standard assumption is that supernova explosions associated with star formation drive galactic winds and reduce the fraction of cold gas that can form stars. In many semi-analytical models of galaxy formation, it is assumed that the mass-loss rate is proportional to the star formation rate, $\dot{M}_{\text {wind }}=\epsilon \dot{M}_{\star}$, where the feedback efficiency $\epsilon$ is assumed to depend on halo circular velocity, $V_{c}, \epsilon=1 /\left[1+\left(V_{c} / V_{c, 0}\right)^{q}\right]$, with $q \sim 2$. We follow Cole et al. (2000) and assume $V_{c, 0}=200 \mathrm{~km} \mathrm{~s}^{-1}$. Note, however, although the feedback model described above has been widely adopted in semi-analytical models, the original model, proposed by Dekel \& Silk (1986), was actually for starburst galaxies, rather than for quiescent disks. Moreover, numerical simulations of star formation in quiescent disks have not been able to provide strong support for these assumptions (e.g. Mac Low \& Ferrara 1999).

We applied the above star formation model to galaxy disks formed in CDM halos. We follow the prescription described in Mo, Mao \& White (1998) to model disks in CDM halos. As a simple model, we assume that all systems have evolved for a sufficiently long time so that the cold gas surface density in each disk is close to the critical density. This 
assumption is supported by the fact that the disks in many spiral galaxies are marginally unstable, with Toomre's $Q$-parameter close to 1 . The mean $M_{\mathrm{HI}}-M$ relation predicted by this model is shown in the left panel of Fig. 2. The predicted $M_{\mathrm{HI}}-M$ relation is much shallower at the low-mass end than that obtained by fitting the observed HI mass function. As shown in the right panel of Fig. 2, this predicted relation, together with the CDM halo mass function, predicts an HI-mass function that is much too steep at the low-mass end.

If we assume that the shape of the halo mass function is similar to that predicted by the standard $\Lambda$ CDM model, the discrepancy between model prediction and observation shown in Fig. 2 must be due to the assumption about how gas settles into the centres of dark halos to form stars. As is well known, the intergalactic medium (IGM) is heated by the UV background after reionisation. Recent simulations show that the fraction of the gas that can be accreted into a dark halo of mass $M$ at the present time can be written roughly as

$$
f_{\text {gas }}=\frac{f_{B}}{1+\left(M_{0} / M\right)^{\alpha}},
$$

where $f_{B}$ is the universal baryon fraction, $M_{0} \sim 10^{10} h^{-1} \mathrm{M}_{\odot}$, and $\alpha \sim 1$ (e.g. Hoeft et al. 2005). We have used this gas fraction together with the star formation prescription described above to predict the cold gas in dark matter halos. The predicted $\bar{M}_{\mathrm{HI}}-M$ relation and HI mass function are shown in Fig. 2. Preheating by a UV background can reduce the HI mass in low-mass halos, but the effect is significant only for halos with masses $<10^{10} h^{-1} \mathrm{M}_{\odot}$ and is not sufficient to explain the observational results.

One interesting aspect of the prediction with the heating of UV background is that the predicted slope of the $\bar{M}_{\mathrm{HI}}-M$ relation at $M<10^{10} h^{-1} \mathrm{M}_{\odot}$ is actually similar to that in the observations. This motivated us to consider a model in which the IGM around low-mass halos is preheated to a temperature that is higher than that of photoionisation heating by the UV background. As a simple model, we assumed that the fraction of gas that can be accreted into dark matter halos has the same form as Eq. 3.1 but with a higher mass scale, $M_{0} \sim 10^{11.5} h^{-1} \mathrm{M}_{\odot}$. As shown in Lu \& Mo (2005, in preparation), the form of Eq. 3.1 is valid for dark halos accreting in a preheated medium, and the mass scale chosen corresponds to an initial specific entropy, $s \equiv T / n^{2 / 3} \sim 10 \mathrm{kev} \mathrm{cm}^{2}$. Fig. 2 shows that the $\bar{M}_{\mathrm{HI}}-M$ relation and the HI-mass function predicted by this model match quite nicely the results of RS, but the predicted $\bar{M}_{\mathrm{HI}}$ mass for a given $M$ is still higher than that required by the Z05 HI-mass function. In order to match the HI-mass function of Z05, we have to assume $M_{0} \sim 10^{12} h^{-1} \mathrm{M}_{\odot}$. Note that in the preheating model considered here, we have assumed that star formation in a halo does not cause any mass loss from the galaxy. In reality, some gas may still be ejected by supernova explosions, which can further reduce the amount of cold gas in small halos.

With the star formation prescription described above, we can also predict the total stellar mass in a halo. Fig. 3 shows the average stellar mass of the central galaxy of a halo as a function of halo mass predicted by the three models described above. The standard model predicts much larger stellar masses for low-mass halos than the other two models. Photoionisation heating by the UV background reduces the stellar masses in small halos, but the effect is not as strong as in the preheating model. Model predictions for $M \gg 10^{11} h^{-1} \mathrm{M}_{\odot}$ are not reliable, because our simple model does not take into account the fact that radiative cooling may become less efficient in large halos, that other feedback effects, such as heating by a central AGN may become important for the more massive halos, and that these halos may contain satellite galaxies. The thick dashed curve shows the relation between the average $b_{J}$-band luminosity and halo mass 


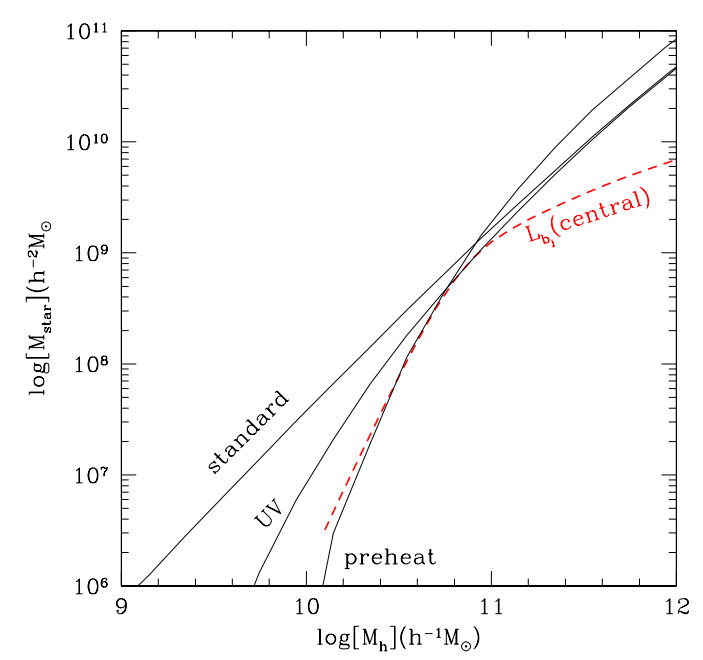

Figure 3. The solid curves show the relations between stellar mass and halo mass predicted by the standard model, the model with heating by the UV background, and the preheating model, respectively. The dashed curve shows the relation between the $b_{j}$-band luminosity of the central galaxy and halo mass, as found in Yang et al. (2005).

recovered by matching the observed luminosity function of galaxies (Yang et al. 2003, 2005). For late type galaxies, the stellar mass-to-light ratio is roughly 1 in the blue band and so the observational result can be compared directly with the model prediction for stellar masses. The standard model clearly predicts too large stellar masses for low mass halos. This result is consistent with the fact that the luminosity functions predicted by such models have too steep faint-end slopes. Photoionisation heating by the UV background helps to reduce the discrepancy, but is not sufficient to reach an agreement. It is interesting that the preheating model, which matches the cold gas mass, can also match the stellar mass. In the preheating model, the stellar mass is reduced because the amount of cold gas available for star formation is reduced by preheating.

All the three models predict that the gas mass fraction decreases with halo mass, in qualitative agreement with observation (e.g. Garnett 2002). This trend is produced because of the existence of the critical surface density and because star formation efficiency is lower in lower mass halos (e.g. van den Bosch 2002). The higher gas mass fraction in smaller halos implies that the metallicity of the cold gas must be lower in lower mass systems, which is consistent with observations (e.g. Garnett 2002). In the standard model, metals are completely mixed with the gas before being ejected from the halo. In this case, the total amount of metals retained by the system is smaller than the amount generated by stellar winds and supernova explosions, so that the effective metal yield is lower than the stellar yield. Since the efficiency of mass loss is higher for halos with lower masses, this model predicts that the effective metal yield decreases with galaxy luminosity, which is consistent with observations (e.g. Garnett 2002; Tremonti et al. 2004). On the other hand, in the preheating model considered above, no outflow is required to match the cold gas mass in low-mass halos. However, this does not exclude the possibility that a significant amount of metals may have been lost from low-mass galaxies without producing a heavy mass loss. It is possible that metals produced by supernova explosions are not well mixed with the cold gas before being ejected. In this case, star formation in a disk still drives an outflow, but the mass in the flow is small and is dominated by the 
metal-rich material from the ejecta. This is indeed what is seen in numerical simulations of supernova-driven galactic winds (e.g. Mac Low \& Ferrara 1999).

\section{Preheating by gravitational pancaking}

In the previous section we have shown that the preheating model, where the gas around present-day low mass halos is preheated to a level $s \sim 10 \mathrm{keV} \mathrm{cm}^{2}$, can help to match the observed HI mass function, as well as the faint end slope of the galaxy luminosity function. Here we describe a physical process that may be responsible for such preheating. The details of the model will be described in Mo et al. (2005).

The model is based on the following considerations. The basic picture of structure formation in the CDM scenario is that, as the Universe expands, larger and larger objects collapse in the cosmic density field due to gravitational instability. The collapse is generically aspherical, first forming sheet-like pancakes and then filamentary structures, and eventually virialised dark matter halos. According to the ellipsoidal collapse model, the formation of a virialised halo requires the collapse of all three axes (e.g. Bond \& Meyer 1996; Sheth, Mo \& Tormen 2001). Because of the presence of large scale tidal fields, the density threshold for the formation of a low-mass halo (i.e. with mass below the characteristic mass $M_{*}$ defined as the mass at which the rms fluctuation is 1) can be much higher than that in the spherical collapse model, and so the formation of such halos is 'delayed' relative to the prediction of the spherical model. On the other hand, the collapse of the first (shortest) axis is accelerated by the tidal field, and so the formation of a pancake requires a density threshold that can be much lower than that for spherical collapse. Consequently, many of the low-mass halos at the present time, i.e. those with $M \ll M_{*}(z=0) \sim 10^{13} h^{-1} \mathrm{M}_{\odot}$, were embedded in pancakes of larger masses that previrialised before the formation of the halos themselves. In the process of pancake formation, the gas associated with pancakes is shocked. If the temperature of the shocked gas is sufficiently high and if the gas is not able to cool in a Hubble time, the halos embedded in the pancakes will accrete gas from a preheated medium, which can suppress the amount of gas that can be accreted. Detailed calculations (Mo et al. 2005) show that the progenitors of present-day low-mass halos $\left(M \lesssim 10^{12} h^{-1} \mathrm{M}_{\odot}\right)$ were embedded in pancakes of masses $\sim 5 \times 10^{12} h^{-1} \mathrm{M}_{\odot}$ at $z \sim 2$. The formation of such pancakes can heat the gas to a temperature of $5 \times 10^{5} \mathrm{~K}$ and compress it to an over-density of $\sim 10$. Such gas has a cooling time longer than the age of the Universe at $z \lesssim 2$, and has a specific entropy comparable to that required to reduce the amount of cold gas and star formation activity in galaxy halos.

\section{Discussion}

In our preheating model, the accretion of gas into low-mass halos is naturally divided into two different regimes. At $z>2$, the IGM to be accreted is cold. Since radiative cooling is efficient in galaxy halos, gas assembly into galaxies is rapid and dominated by cold gas clumps. Combined with the fact that the formation of galaxy-sized dark matter halos at $z \gtrsim 2$ is dominated by major mergers, this suggests that during this period of time gas can collapse into halo centres quickly to form starbursts and perhaps also to feed active galaxy nuclei. Galaxy winds associated with such systems may drive strong winds into the IGM, contaminating the IGM with metals and perhaps also heating the medium. At $z \lesssim 2$, however, the situation is quite different. Since the medium in which present-day low-mass halos formed was already heated up by gravitational pancaking, and radiative cooling was no longer efficient, the accretion is dominated by hot, diffuse 
gas. This gentle gas accretion is likely responsible for the formation of the quiescent disks of spiral galaxies.

The preheated medium we are envisaging here is closely connected to the warm-hot intergalactic medium (WHIGM) which has been under intensive discussion in recent years (see Cen \& Ostriker 1999, Dave et al. 2001 and references therein for simulation results; see Tripp et al. 2005 for a review of the related observations). Hydrodynamical simulations clearly show that the WHIGM is produced by shocks associated with gravitational collapse in the cosmic density field. Our results demonstrate that such a medium may have significant consequences for the formation of late-type galaxies, because the formation of dark halos in the cosmic density field is such that many of the low-mass halos at the present time may have assembled their gas after the medium around them has been heated up by gravitational pancaking. Clearly, further investigations with sophisticated models and numerical simulations are required in order to quantify in detail the relationship between galaxy halos and their environment, and how preheating by gravitational collapse can affect the formation of galaxies in the cosmic density field.

\section{Acknowledgements}

We thank Jessica Rosenberg and Martin Zwaan for providing us with their HI mass functions.

\section{References}

Benson, A. J., Bower, R. G., Frenk, C. S., Lacey, C. G., Baugh, C. M., Cole, S., 2003, ApJ, 599, 38

Bond, J. R., Myers, S. T., 1996, ApJS, 103, 1

Cen, R., Ostriker, J. P., 1999, ApJ, 514, 1

Cole, C., Lacey, C. G., Baugh, C. M., Frank, C. S., 2000, MNRAS, 319168

Dave, R., et al., 2001, ApJ, 552, 473

Dekel, A., Silk, J., 1986, ApJ, 303, 39

Garnett, D. R., 2002, ApJ, 581, 1019

Gnedin, N. Y., 2000, ApJ, 542, 535

Hoeft, M., Yepes, G., Gottloeber, S., Springel, V., 2005, preprint (astro-ph/0501304)

Kang, X., Jing, Y. P., Mo, H. J., Börner, G., 2004, submitted to ApJ, preprint (astro$\mathrm{ph} / 0408475)$

Kennicutt, R. C., 1989, ApJ, 344, 685

Kennicutt, R. C., 1998, ApJ, 498, 541

MacLow, M. M., Ferrara, A., 1999, ApJ, 513, 142

Mo, H. J., Mao, S., White, S. D. M., 1998, MNRAS, 295, 319

Mo, H. J., Yang, X., van den Bosch, F. C., Katz, N. S., 2005, to be submitted

Rosenberg, J. L., Schneider, S. E., 2002, ApJ, 567, 247 (RS)

Sheth, R. K., Mo, H. J., Tormen, G., 2001, MNRAS, 323, 1

Tremonti, C. A., et al., 2004, ApJ, 613, 898

Tripp, T., Bowen, D. V., Sembach, K. R., Jenkins, E. B., Savage, B. D., Richter, P., 2004, astro-ph/0411151

van den Bosch, F. C., 2002, MNRAS, 332, 456

van den Bosch, F. C., Yang, X. H., Mo, H. J., 2003, MNRAS, 340, 771

van den Bosch, F. C., Yang, X. H., Mo, H. J., Norberg, P., 2005, MNRAS, 356, 1233

White, S. D. M., Rees, M. J., 1978, MNRAS, 183, 341

Yang, X. H., Mo, H. J., Jing, Y. P., van den Bosch, F. C., 2005, MNRAS, 358, 217

Yang, X. H., Mo, H. J., van den Bosch, F. C., 2003, MNRAS, 339, 1057

Zwaan, M. A., et al., 2003, AJ, 125, 2842

Zwaan, M. A., Meyer, M. J., Staveley-Smith, L., Webster, R. L., 2005, astro-ph/0502257 (Z05) 